\title{
XL-WMSN: cross-layer quality of service protocol for wireless multimedia sensor networks
}

\author{
Zara Hamid ${ }^{*}$ and Faisal Bashir
}

\begin{abstract}
Energy conservation has been the prime motivation behind the design of conventional protocols for wireless sensor networks (WSNs). However, recent trends toward high data rate multimedia communication over WSNs demand traffic- and deadline-aware content delivery with minimum energy expenditure. The basic quality of service requirement in wireless multimedia sensor networks (WMSNs) is time-bound data delivery. The conventional-layered protocol design solutions are inefficient, as real-time content delivery requires interactions between multiple layers like application for traffic categorization, network for real-time delivery, and media access control (MAC) for prioritized medium access with minimum energy expenditure. In this paper a cross-layer solution (XL-WMSN) is proposed for real-time data delivery. The XL-WMSN provides interaction between energy-based admission control, delay- and interference-aware routing, and dynamic duty cycle assignment at MAC layer. Simulation analysis shows that XL-WMSN increases the probability of delivering multimedia content within their allocated deadline and is more efficient than existing solutions.
\end{abstract}

\section{Introduction}

The recent shift in wireless communication paradigm towards real-time multimedia communication has led to a burgeoning of new concepts and designs. This evergrowing attention towards multimedia communications has also influenced innovations in the design of wireless sensor networks, leading to the birth of WMSNs. These are distributed wireless networks of heterogeneous nodes (consisting of scalar, audio, and video nodes) that gather important information about their physical environment. Lately, a plethora of applications [1-3] are taking advantage of WMSNs to help make more informed decisions.

There are several applications that can significantly benefit from WMSNs. In particular, these networks are most relevant for surveillance and monitoring applications for military, agriculture, health care, disaster relief, etc. With the increase in all kinds of security and terrorism threats to society and economic assets, research towards efficient design of these applications is a necessity. However, the nature and volume of information acquired in these

*Correspondence: zara@mcs.edu.pk

Computer Software Engineering, College of Signals, National University of Sciences and Technology (NUST), Islamabad, Pakistan applications present as a great challenge for resourcedeficient sensor networks. Heterogeneous traffic flows in surveillance applications demand latency-aware protocol that incorporate service differentiation to meet strict end-to-end deadlines. In addition, media access control (MAC) protocol that can provide prioritized medium access according to traffic type is a requirement in a surveillance application.

These networks demand stringent quality of service (QoS) guarantees like delivery of data within deadline, minimizing distortion and jitter, reliability, and high throughput. But meeting end-to-end delay deadline is the most important design parameter for delay-constrained multimedia communications. The latency at all layers needs to be minimized to achieve the end-to-end delay guarantees. Particularly, the network and MAC layer have a huge role in minimizing end-to-end latency. End-to-end delay can be reduced at the network layer by choosing the shortest path or path with minimum delay. There exist several routing protocols which focus on minimizing latency. This [4] protocol incorporates the channel delay of the link including queuing delay, propagation delay and protocol processing time, available bandwidth, and cost of

\section{至 Springer}

(c) 2013 Hamid and Bashir; licensee Springer. This is an Open Access article distributed under the terms of the Creative Commons Attribution License (http://creativecommons.org/licenses/by/2.0), which permits unrestricted use, distribution, and reproduction in any medium, provided the original work is properly cited. 
each path to choose the least delayed path. Several routing protocols aim to achieve delay deadlines of packets by transmitting them at a certain speed. These provide service differentiation as well as end-to-end delay guarantees [5-7]. Authors in [5] calculate the velocity requirement according to the delay at each forwarding node and choose the most energy-efficient neighbor that meets the velocity requirement. If the velocity requirement cannot be met by any neighbor, then the power level of the node is adjusted to meet the delay constraints. Akkaya and Younis [8] provided differentiated service according to real-time and non-real-time traffic flows. This protocol guarantees energy-efficient paths that meet end-to-end delay requirements.

Delay at MAC layer can be minimized by using a prioritized scheduling scheme which gives priority to delay intolerant applications. RAP [9] is a communication architecture for sensor networks that proposes velocitymonotonic scheduling in order to minimize deadline miss ratios for packets. Each packet is put to a different FIFO queue based on their requested velocity, i.e., the deadline and closeness to the gateway. This ensures prioritization at the MAC layer. In [5-8] traditional layered approach is used to provide different solutions for routing, MAC, and rate control, where each layer provides service only to its adjacent higher layer. This provides modularity and transparency between layers which leads to robust protocol design. However, layered architecture provides a suboptimal solution for WMSNs for two main reasons: (a) limited interaction between adjacent layers through well-defined interfaces, e.g., MAC layer in a layered approach cannot provide channel utilization information to transport layer for rate adjustment and (b) individual processing time per layer increases due to lack of interaction among different layers. Accordingly, cross-layer design has emerged as a promising approach for efficiently meeting multi-constrained requirements of WMSNs. WMSNs by nature have interdependence between different layers of the protocol stack. This interdependence can be exploited, through crosslayer cooperation, to guarantee application-specific QoS constraints.

This paper proposes a cross-layer protocol that incorporates energy-aware admission control along with delay- and traffic-aware routing protocol and an endto-end deadline-aware duty cycle to provide delayconstrained delivery of multimedia data while conserving energy.

The remainder of the paper is organized as follows. In Section 2, we describe and discuss some of the related work. The XL-WMSN basics and detailed description is provided in Section 3. In Section 4 we present the performance analysis and results and finally the conclusion is given in Section 5.

\section{Related work}

A brief survey of recent literature reveals that there is a growing trend towards cross-layer schemes which focus on achieving delay-constrained delivery of multimedia data along with energy minimization. Several cross-layer schemes have been proposed for WMSNs. In this section, we focus on schemes in which MAC and network layer jointly provide real-time routing and prioritized medium access. A detailed survey on cross-layer protocols for WMSNs is given in [10].

Sensor medium-access control (SMAC) [11] protocol is the earliest and most straight-forward, duty cycle-based MAC protocol that incorporates local synchronization and static sleep and listen cycles. It is a simple carrier sense multiple access (CSMA)-based protocol, which conserves node energy at the cost of increase in latency and decrease in throughput. T-MAC is proposed to enhance the poor results of the SMAC protocol under variable traffic load. TMAC [12] is a traffic-adaptive duty cycle assignment scheme for sensor networks. It is an improvement over SMAC and saves more energy under variable load as compared to SMAC. TMAC uses a time-out value (TA) to determine the end of an active period. A node performs channel sensing during TA interval; if it does not overhear any communication during this interval, it safely concludes that no neighbor wants to communicate and goes to sleep. Otherwise, it refreshes its TA interval. The downside to TMAC's traffic adaptive mechanism is that nodes go to sleep early, resulting in low throughput and high latency.

Saxena et al. [13] propose a MAC layer protocol based on CSMA-CA approach which aims to provide QoS for multimedia communications over sensor networks while conserving energy. This protocol provides differentiated service to each traffic class through dynamic adjustment of contention window (CW) size for each class. In this protocol, CW of a low-priority traffic class increases faster and reduces slowly, whereas $\mathrm{CW}$ of high-priority traffic class increases slowly and reduces faster. Energy conservation is provided by adjusting the duty cycle according to the dominantly processed traffic on nodes. The duty cycle is pre-defined for each traffic class and uniform across the nodes.

In [14] authors propose an interaction between application, network, MAC, and link layer to maximize the number of video flows while keeping the overall distortion below threshold. The application layer determines the end-to-end delay and coding rate; at network layer a multipath routing algorithm is used to determine routes that can provide end-to-end delay and bandwidth guarantees. In case the relaying node is unable to meet the bandwidth, it performs link adaptation algorithm to switch to a higher transmission rate; if a higher transmission rate is not available, then a reject message is sent to the node. In case none 
of the available paths can provide the available bandwidth, the source increases its GoP size. At MAC layer, IEEE 802.11e framework is used to provide prioritized access.

The cross-layer control unit (XLCU) [15] relies on an integrated MAC and physical layer based on ultra wide band. XLCU provides joint cooperation among application, network, MAC, and physical layers and also provides admission control functionality based on hopby-hop contracts. These hop-by-hop contracts are guaranteed through packet-level service differentiation at each hop in terms of throughput, end-to-end delay, and end-toend packet error rate. The use of UWB technology allows an integrated MAC and physical layer which removes the need for mutual exclusion since simultaneous transmission is possible through the use of different time hopping sequences by each sender. However, collisions can occur at the receiver, which is solved in the protocol by using a receiver-centric scheduling algorithm. XLCU also provides dynamic channel coding to adapt to the level of interference at the receiver. XLCU covers the issue of providing end-to-end QoS guarantees and resource management but does not involve transport layer to provide rate control.

In [16] a geographic routing protocol for multipath routing of real-time video frames is proposed. It uses an intelligent scheduling scheme, which maintains a table of each active path's delay, available bandwidth, etc., and sends each video frame on the most suitable path according to its priority and delay requirements. In case a suitable path is not available, i.e., insufficient aggregate bandwidth, the video frame is dropped and the application is sent a message to reduce the coding rate. By using hop-by-hop deviation angle adjustment method, a path can be established using any initial deviation angle specified at the source node, and then other disjoint paths are constructed by changing the value of the deviation angle. However, like most geographical routing protocols, the proposed cross-layer design assumes that nodes are location-aware and that the density of nodes is high.

PMAC [17] presents a cross-layer duty cycle MAC protocol which divides the network region into different grades, depending on each region's advancement to the sink. Consequently, there is a different sleep/wake schedule for nodes in each grade. PMAC ensures that the nodes maintain staggered schedules between any two adjacent grades and ensures that the data are forwarded from source to sink in a pipeline fashion. Although network layer is integrated with the duty cycle scheme to support the transmission of packet from a node in one grade to another (in a pipeline manner), PMAC provides no mechanism for efficient and latency-aware route establishment. Thus, the performance of PMAC is dependent on the underlying routing scheme.
Our previous work on delay- and channel utilizationaware routing has been presented (Z Hamid, J Y Pyun, and F Bashir, unpublished work) and in [18]. This paper presents detailed discussion and analysis on the relationship between channel utilization and delay and its impact on the routing decision. Moreover, in this work, a thorough performance analysis of the routing mechanism is carried out for organized network topology.

\section{XL-WMSN: cross-layer protocol for WMSNs}

The design principle of XL-WMSN is a joint crosslayering between the network and MAC layer, such that both layers exchange information and support each other in efficiently achieving delay deadlines. The different functionalities of these layers that are considered in the unified cross-layer model are explained in the following sections.

\subsection{Network model}

In this section, basic system definitions, assumptions, and principles of XL-WMSN are presented. The sensor network is represented as a graph $G(V, E)$, where $V=$ $\left\{v_{i}, \ldots, v_{N}\right\}$ is a finite set of nodes in a finite dimension terrain, with $N=|V|$, and $E$ is the set of links among nodes, i.e., $e_{i j} \in E$ iff nodes $v_{i}$ and $v_{j}$ are within each others transmission range. Node $v_{N}$ represents the sink. We assume $N$ as heterogeneous sensor nodes based on the type of data they generate. Sensor nodes are distributed in a grid-like arrangement in the sensing field. However, for simplicity, all nodes have the same transmission range and initial energy value and are considered to be non-mobile. The network is fully connected and each node is willing to participate in communication process. Nodes forward data to sink in a multi-hop fashion, forming many-to-one routing paradigm.

Each node performs distributed duty cycle operation such that the node is switched on for a certain fraction of the time and is switched off for the remaining fraction of the time. It is assumed that the sink is a special node, which is always switched on. The on-off periods are managed through a duty cycle parameter, $\delta$, which defines the fraction of the time when a node is active. Nodes have the same duty cycle at the time of deployment. However, their duty cycle is not synchronized with each other.

The communication channel is assumed to be errorfree. The shared channel access is assumed for network nodes using CSMA-based methodology. The carrier sensing range of each node is denoted by $d_{\mathrm{s}}$, where $d_{\mathrm{s}} \geq d_{\mathrm{c}}$, i.e., the carrier sensing range is larger than or equal to the communication range. Whenever a node receives two or more packets sent by nodes within its carrier sensing range, all of them are lost. On the other hand, if a node senses a single packet, it can always receive it successfully, since we assume an error-free channel. We assume a connected network of sensor nodes; each sensor receives 
and transmits packets in a multi-hop fashion. Received packets are stored at the incoming buffer and queued for service by the MAC layer.

WMSNs are event-based networks with heterogeneous flows. Each traffic flow has different end-to-end delay requirements, particularly, video traffic has strict latency requirements. The network is composed of $m$ traffic flows, $n$ sources which exist in the vicinity of an event area, some relay nodes, and a single sink. Furthermore, multiple flows can be flowing through the same node.

\subsection{Energy-aware admission control}

Admission control is an important mechanism used for QoS provisioning in a network. It ensures that a new traffic flow is allowed only if there are sufficient resources in the network to meet the QoS requirements without violating the QoS of already accepted requests. There exist several admission control schemes, which handle admission control with respect to different QoS factors, such as energy consumption, resource utilization, or feasibility. CACP [19] bases its decision on the level of contention between neighbors. It calculates whether the available bandwidth is sufficient for the new flow. The proportional distribution admission control [20] scheme provides a network layer-based, prioritized distributed admission control and bandwidth reservation algorithm that allows a mobile node to establish or discard a flow based on the traffic flow's priority, channel conditions, transmission rates, and interference on link, etc. In [21] an admission control scheme with multi-constrained QoS providing is proposed. This scheme treats different packets differently according to their delay and reliability constraints, giving higher priority to real-time traffic.

XL-WMSN aims to provide QoS guarantees along with energy minimization. To achieve this goal, the proposed admission control scheme takes into consideration the remaining energy on a node. By considering energy in admission control decision, XL-WMSN helps lesser energy nodes keep out of routing paths and thus avoid battery drainage which results into network holes.

To reduce message overhead, XL-WMSN implements admission control during the route discovery process to eliminate nodes without enough energy. When a source node has data to send, it broadcasts a route request (RREQ) packet to its neighbors. Each node that receives the RREQ determines whether it wants to participate based on the value of $P$. The value of $P$ is determined as follows:

$$
P= \begin{cases}1 & E_{\text {Rem }}>E_{T} \\ 0 & \text { otherwise }\end{cases}
$$

The value of $P$ is 1 if remaining energy of node, $E_{\text {Rem }}$, is greater than a threshold, $E_{\mathrm{T}}$, in which case the node is partially admitted in the route establishment, and its weight is calculated next (as discussed in Section 3.3) to determine its suitability as compared to its neighbors. When the sink receives the RREQ and sends a RREP, the partial route in the RREQ becomes a full route. In case $P$ is 0 , the RREQ is simply dropped.

\subsection{Delay-aware routing}

Identification of appropriate path selection metrics is the biggest design challenge of any routing protocol. In WMSNs, achieving high throughput, low latency and jitter, along with low-path setup time is imperative. Therefore, XL-WMSN uses a combination of average packet service time $\mathrm{PST}_{\mathrm{avg}}$, channel utilization Util ${ }_{i}$ and hop count in its routing decision. Packet Service Time (PST) provides information of load on a node, while channel utilization is a good indicator of local contention. Hop count is used as a mechanism to restrict the path length since an increasing path length leads to decrease in the probability of successful delivery of information. In the remaining of this section, the above-mentioned parameters are explained with regard to their utility in XL-WMSN protocol.

\subsubsection{Packet service time}

Several factors contribute to the overall delay of packets at a node including queuing, network layer, MAC layer, and transmission delays:

1. Queuing delay. This is the time a packet spends waiting in a queue before it reaches the head of the queue. The difference between the outgoing packet rate and the incoming packet rate determines the queuing delay. In high data rate multimedia sensor networks, links are easily congested, which leads to high contention that can reduce the outgoing rate of a node. This leads to high queuing delays, which indicate that the node has a high load and it should be restricted from participating in any fresh route establishment.

2. Network layer delay. This includes all the processing delay involved in path determination and forwarding of packet to lower layers.

3. MAC layer delay. This takes into account all delays due to contention, such as channel sensing and channel reservation.

4. Transmission delay. This delay depends on the packet size and bandwidth. XL-WMSN takes into consideration all the above-mentioned delays to calculate the PST of a node.

The PST is expressed as follows:

$$
T_{\mathrm{PST}}=T_{\text {Net }}+T_{\text {Queue }}+T_{\mathrm{MAC}}+T_{\text {Transmit }},
$$


where $T_{\mathrm{NET}}$ is the network processing delay, $T_{\text {Queue }}$ is the queuing delay, $T_{\mathrm{MAC}}$ is the MAC layer delay, and $T_{\text {Transmit }}$ is the transmission delay.

By monitoring the PST of each packet during every period of time, $t$, the average packet service time, PST $_{\mathrm{AVG}}$, can be estimated using a weighted moving average as given in Algorithm 1, where $\beta(0 \leq \beta \leq 1)$ is a constant value used to assign weights to current $\mathrm{PST}_{\mathrm{AVG}}$ measurements with respect to past measurements, thus smoothing the consequences of past trends. Assigning higher weight to $\beta$ gives more preference to latest PST AVG value observed in the current interval as compared to previous. Thus, in dynamic and unpredictable WMSNs, where sudden burst of data can suddenly increase congestion and contention, assigning higher weights to more recent observations can make routing more robust and responsive to abrupt changes.

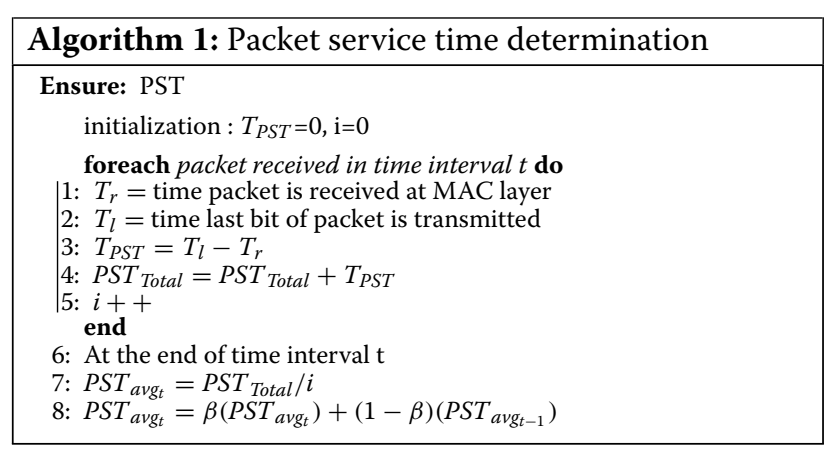

\subsubsection{Channel utilization}

The average PST is a good indicator of delay and congestion around a node. Therefore, this metric can be considered sufficient for choosing most efficient routes. However, in wireless networks the transmission medium is shared and communication from one node may consume the bandwidth and consequently affect the channel utilization of neighboring nodes. Thus, it is possible for a node which is not participating in transmission or relay of packets itself to have high contention delay due to active neighboring nodes. If routing decision is made on PST alone, then idle nodes in high contention area will also be considered as potential relaying nodes, since their PST will be zero. Whereas in reality, these nodes should be considered as highly unfavorable since they will have high channel access delay once the transmission starts.

Measuring channel utilization is an energy-intensive task as it requires a node to be awake and listen to the channel at regular intervals. Since sensor nodes are energy-constrained, it makes sense to perform channel utilization only on nodes which detect a busy channel.
We modify the MAC layer in a way that busy nodes perform channel utilization at regular intervals, whereas nodes that do not detect the channel as busy perform channel utilization after longer intervals. In case of idle nodes, the interval is incremented by value equal to short interframe space (SIFS) duration, which is $16 \mu$ s. This results in a more energy-efficient utilization of resources. Algorithm 2 shows how often a channel utilization module is called for active and idle nodes. Channel utilization is performed at regular intervals $t$ for active nodes, and the weighted moving average is performed after a fixed interval $T$; whereas $t$ is incremented for idle nodes each time the node is found to be idle until it grows as big as $T$, after which channel utilization is performed only once at the start of each interval $T$.

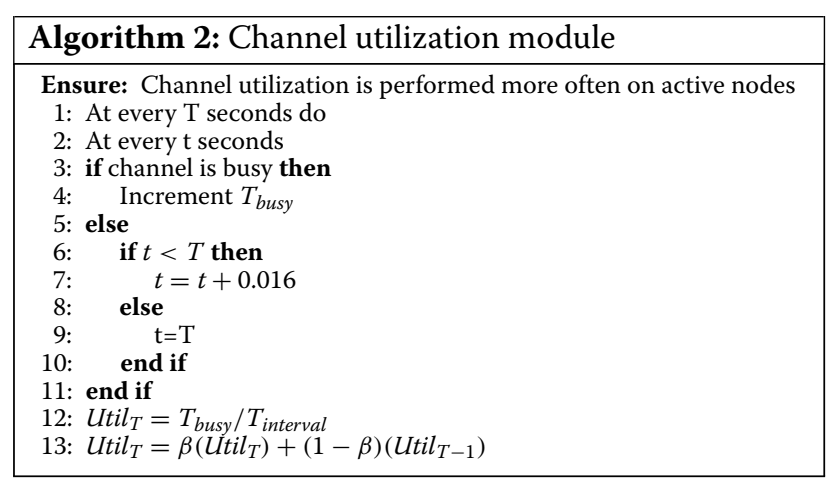

\subsubsection{Path establishment}

XL-WMSN uses a reactive approach for routing where path is established only if a node has data to send. Reactive approach lowers the overhead of control messages; therefore, it is proper for energy-constrained sensor networks. The operation of the routing protocol depends on how a node calculates its own weight. Each node calculates its weight based on PST $_{\mathrm{AVG}}$ and Util, using the equation below:

$$
W_{i}=\alpha\left(1-\operatorname{PST}_{\mathrm{vg}_{i}}\right)+\gamma\left(1-\mathrm{Util}_{i}\right),
$$

where $W_{i}$ is the node's own contribution to the total cost of the path, $\mathrm{PST}_{\mathrm{AVG}}$ represents the average PST, and Util represents the channel utilization at time instance $i$. The $\alpha$ and $\gamma$ are the coefficients that assign weight to the associated factors, where $\gamma=(1-\alpha)$. The value of these coefficients can vary from 0 to 1 and have been determined through extensive simulations and discussed in Section 4.

When an event occurs, the source nodes broadcast RREQ messages to find a least delayed path to the destination. During path establishment each node receives multiple RREQ messages and establishes reverse route with the node that has the highest weight. The RREQ 
packet contains the source ID of the packet, broadcast ID, number of hops the packet has traveled (HopCnt Prev $_{\text {) }}$, previous node's weight ( $\left.W_{\text {Prev }}\right)$, and previous node's id (ID Prev).

As mentioned in Algorithm 3, each node after receiving a RREQ first checks whether it has already received the same request or if it is a new request. If it is receiving a RREQ for the first time, it will add the source node's id, $W_{\text {Prev }}$, HopCnt Prev $_{\text {to }}$ to the routing table and broadcast the RREQ packet, replacing its own node id, $\mathrm{ID}_{N}$ and weight, $W_{N}$ in the RREQ packet. If it is a duplicate RREQ, it compares the weight, $W_{\text {Prev }}$, in the receiving RREQ packet with the weight, $W_{T}$, in its routing table and the difference in hops of the previous entry in the routing table, HopCnt $_{T}$, with the hop count value in duplicate RREQ,

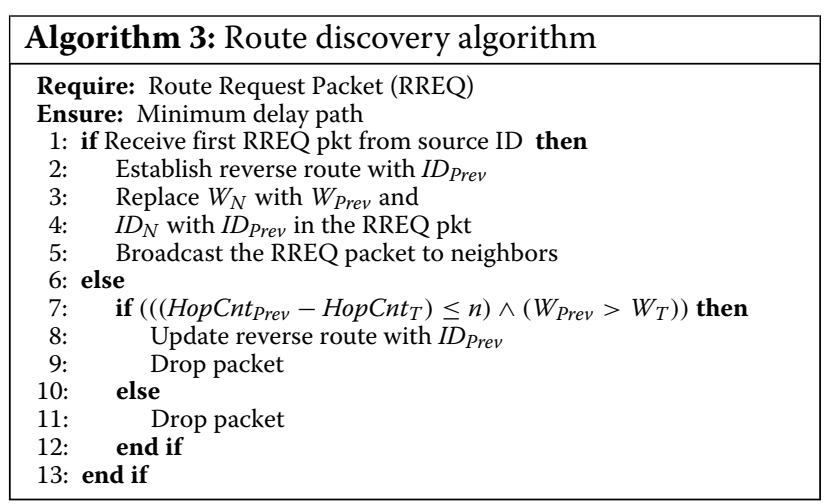

HopCnt $t_{\text {Prev }}$. If the $W_{\text {Prev }}$ value, is greater than $W_{T}$ and the difference in hop count is less than equal to $n$, then it establishes a reverse route with the node that has sent the duplicate RREQ packet and drops the RREQ packet without broadcasting it further. Otherwise, it simply drops the packet. By allowing the nodes to deviate from the minimum path by a maximum of $n$ hops, XL-WMSN provides leverage to allow unused nodes with lesser PST and contention. While this increases the number of transmissions, it also increases throughput and reduces congestion in the network. The value of $n$ depends on node density and has been determined through simulations presented in Section 4.

\subsection{Adaptive MAC}

There is need to design an energy-efficient MAC protocol which is highly responsive to changing network conditions and provides low latency for packet delivery as well as high throughput. Periodically putting the radios of WSN devices into sleep has been widely recognized as the most effective way of saving energy in WSNs [22]. In order to optimize the networks performance, various sleep/wake schemes have been proposed, which consist of schemes employing pre-defined duty cycle, differential duty cycles, and adaptive duty cycles. Pre-defined duty cycle schemes result in high-energy wastage due to idle listening, low throughput, and high latency due to unawareness of underlying network conditions [23]. Thus, static duty cycle schemes are not suitable for WMSNs, which mandate high throughput and low latency. Adaptive schemes utilize different metrics, such as traffic priority, traffic load, residual energy, network topology, and sensor density to adjust duty cycle on nodes. Majority of the duty cycle schemes aim to conserve energy and increase network lifetime in sensor networks, but the integration of multimedia data with sensor networks has led to a requirement for high throughput and low latency duty cycle schemes. In this work we discuss a dynamic traffic-aware duty cycle which takes into consideration PST, traffic priority, and per-hop delay requirement.

\subsubsection{Traffic classification and queuing model}

In this work, traffic types are classified as

- Class 0. This class represents delay intolerant traffic, which has strict end-to-end delay deadlines, such as video traffic from camera in a surveillance application. The video traffic is assumed to be encoded with H.264/AVC codec with $176 \times 144$ pixel resolution. Each video frame has a size of 1,024 bytes and is sent at a constant arrival rate of 30 frames/s.

- Class 1. This class represents delay-tolerant traffic which has relaxed end-to-end deadlines. Acoustic, image, and PIR data are examples of such traffic. Class 1 traffic is modeled as packets having a size of 500 bytes and sent at the same arrival rate as class 0 .

- Class 2. This consists of broadcast and route update or synchronization traffic. Each packet is assumed to have a size of 200 bytes and arrival rate of 20 packets/s.

Class 0 traffic is assigned the highest priority in XLWMSN; class 1 traffic with less strict delay bounds is the second highest priority, and class 2 traffic is the least priority traffic. Packets from each traffic type are buffered in separate queues. Figure 1 illustrates the queuing model used in this work.

Each node is equipped with a packet classifier which classifies the incoming packet according to its priority and assigns it to the appropriate queue. Each queue has a different backoff exponent. In case of virtual collision, packets from higher priority queue is scheduled for transmission. Provisioning multiple queues does not increase the memory overhead considerably, since the queuing model is used; we just split the different packet types in the separate queues instead of storing those in a single queue. 


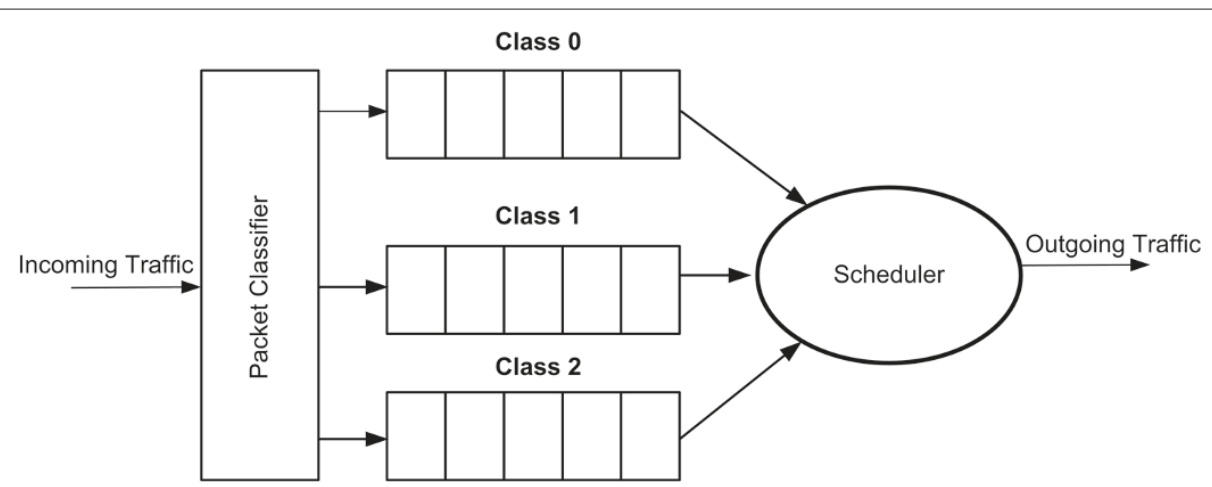

Figure 1 Queuing model.

A multi-queue system can lead to unfairness among different priority queues, resulting in starvation of packets in lesser priority queues. Many of the protocols apply explicit prioritization (due to its simple implementation) to always serve higher priority queues first. In order to provide some level of fairness, the proposed protocol dynamically changes the priority of packets in lower priority queues. When a packet spends a certain amount of time in a lower priority queue, its priority is automatically upgraded, after which it is moved one level higher to a higher priority queue. To further facilitate fairness and QoS provisioning in the queuing scheme, the packets are arranged within a queue according to remaining time to deadline first.

\subsubsection{Duty cycle assignment}

In this section, the operation of dynamic duty cycle assignment (DCA) is discussed in detail. Sensor nodes experience varying traffic loads, so, naturally, for nodes experiencing mainly class 0 traffic (with strict delay deadline), a longer duty cycle is expected with more energy consumption; whereas for nodes experiencing mostly class 1 and 2 traffic more idle time is expected, thus sacrificing throughput and end-to-end latency. Therefore, the goal of DCA is to vary duty cycle according to underlying network conditions and dominant traffic class-specific deadline. DCA achieves this goal by utilizing normalized values of delay and incoming traffic rate and dominant traffic class information. Each of these parameters as well as the detailed working of DCA is described below.

At every time interval, $t$, DCA performs several steps. It measures the dominant traffic flow to determine the traffic-dependent-per-hop delay deadline, $d_{\text {hop }}$. It also calculates the observed delay, $d_{i}$, which is the average PST value on nodes. The normalized measure of delay, $\eta_{i}$, with respect to $d_{\text {hop }}$, at time instance $i$, is calculated as follows:

$$
\eta_{i}=\frac{d_{i}}{d_{\mathrm{hop}}}
$$

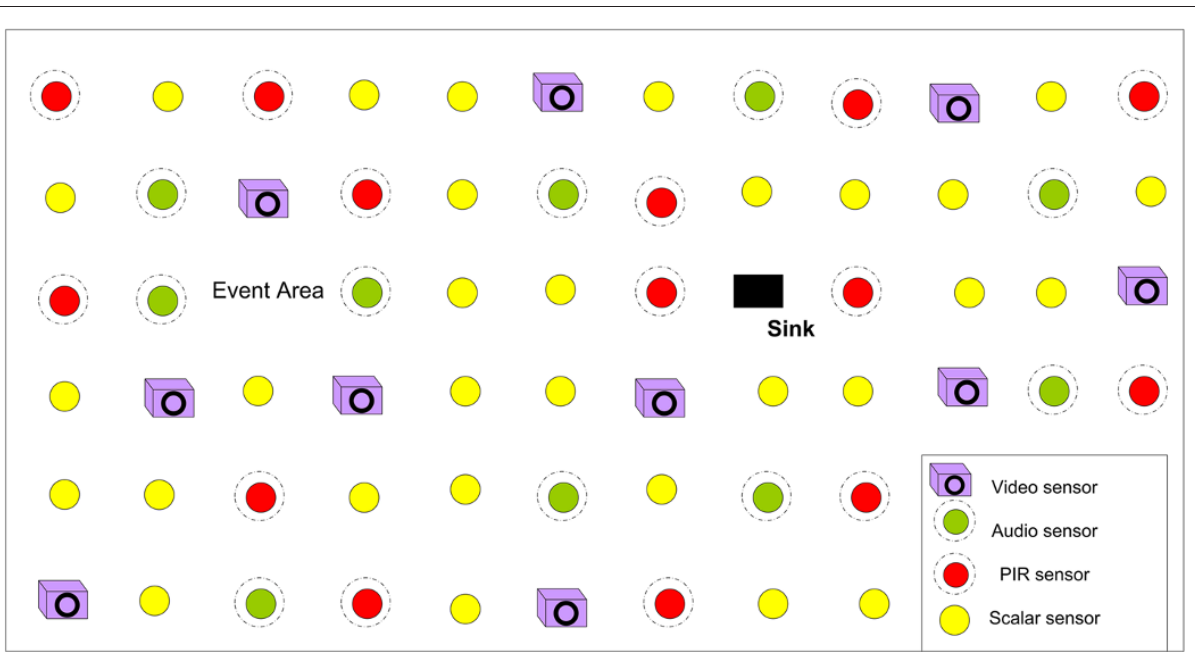

Figure 2 Simulation scenario. 
The per-hop delay requirement is calculated by dividing the end-to-end delay deadline according to the observed packet service time at each hop. The allowed per-hop delay should be less where packet service time is low and high where packet service time is high. We break the endto-end delay deadline into a single-hop delay deadline on each hop as

$$
d_{\text {hop }}=\frac{\operatorname{PST}(r)}{\sum_{r \in p} \operatorname{PST}(r)} \times D_{\mathrm{dl}}
$$

where $\operatorname{PST}(r)$ is the average packet service time at node $\mathrm{r}, \sum_{r \in p} \operatorname{PST}(r)$ is the cumulative PST values of nodes on a particular path to the sink and $D_{\mathrm{dl}}$ is the traffic classspecific, end-to-end delay deadline. DCA also considers traffic rate into its duty cycle update decision. The normalized value of traffic rate at a node, $\tau_{i}$, at time instance $i$, is measured as

$$
\tau_{i}=\frac{\lambda_{i}^{n}}{\lambda_{i}^{\operatorname{sink}}}
$$

where $\lambda_{i}^{n}$, is the average traffic rate at node $\mathrm{n}$ at time instance $i$, and $\lambda_{i}^{\text {sink }}$ is the average traffic rate at the sink at time instance $i$.

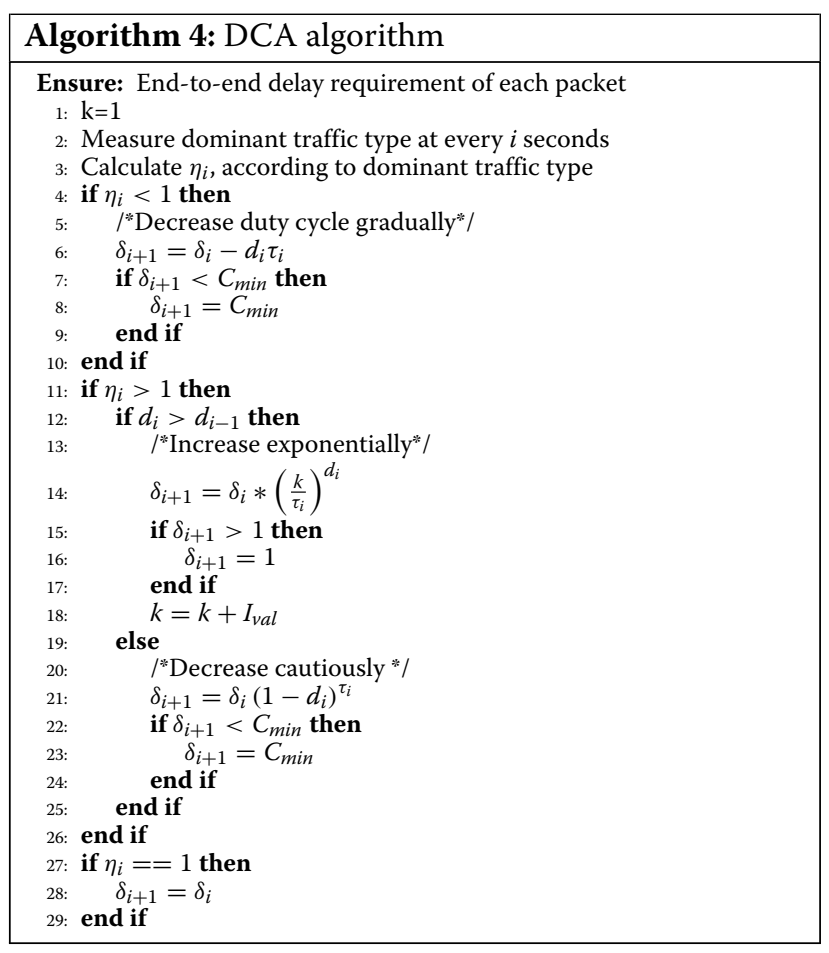

The DCA algorithm strives to keep the value of $\eta$ close to 1 to maintain a balance between energy and latency. The value of $\eta$ is calculated at every $i$ seconds. There are three possible values of $\eta$ :
1. $(\eta<1)$. A value of $\eta$, less than 1 , means that the observed per-hop delay is less than the desired per-hop delay. This can be the result of one or more of the following:

- The particular node is far from the sink where the traffic load is low.

- The node is participating in the transmission or relay of class 1 or 2 traffic which have low data rate.

In this situation, the duty cycle (denoted by $\delta_{i}$ ) can be safely decreased to conserve node energy without compromising on end-to-end delay requirement. However, decreasing the duty cycle aggressively may result in decrease in throughput as well as a sudden rise in PST value. Therefore, the duty cycle must be decreased gradually to allow the value of $\eta$ to converge gracefully and to observe the effect of change in duty cycle on the average PST. This prompts the use of the following linear decrease strategy to update the duty cycle:

$$
\delta_{i+1}=\delta_{i}-d_{i} \tau_{i} .
$$

Conversely, the duty cycle can only be decreased to $C_{\min }$, which is the minimum permissible duty cycle value.

2. $(\eta>1)$. When the value of $\eta$ is greater than 1 , it indicates that the observed delay is greater than per-hop delay constraint. This can be due to the following reasons:

- The particular node is close to the sink where the traffic load is high.

- The node is participating in the transmission or relay of class 0 traffic which has high data rate.

- The node exists in a high contention area, resulting in high channel access delay and packet drops.

In this situation, two cases can occur:

(a) $\left(d_{i}>d_{i-1}\right)$. The observed delay in the decision interval $i$ is greater than delay in interval $i-1$, which shows that the delay is continuously rising. This urges the need to immediately control the sharp rise in delay, which encourages the use of following exponential increase strategy:

$$
\delta_{i+1}=\delta_{i} *\left(\frac{k}{\tau_{i}}\right)^{d_{i}}
$$

The variable $k$ is updated according to a constant value. 
Table 1 Networking parameters

\begin{tabular}{|c|c|c|c|}
\hline Parameter & Value & Parameter & Value \\
\hline Class (data rate) & $\begin{array}{l}\text { Class } 0(120 \mathrm{~KB} / \mathrm{s}) \text { Class } 1 \\
(43.9 \mathrm{~KB} / \mathrm{s}) \text { Class } 2(11.7 \\
\mathrm{KB} / \mathrm{s})\end{array}$ & Radio range & $20 \mathrm{~m}$ \\
\hline Packet size & $\begin{array}{l}\text { Class } 0 \text { ( } 1 \text { KB) Class } 1 \text { (500 } \\
\text { bytes) Class } 2 \text { (200 bytes) }\end{array}$ & Network layer & $\begin{array}{l}\text { AODV/delay-aware } \\
\text { routing protocol }\end{array}$ \\
\hline \multirow[t]{2}{*}{ Re-tx limit } & 4 & MAC & $\begin{array}{l}\text { Deadline-aware } \\
\text { MAC/SMAC/TMAC/ }\end{array}$ \\
\hline & & & Saxena/PMAC \\
\hline$C W_{\min }$ & 7 & Receive power & $22.2 \mathrm{~mW}$ \\
\hline$C W_{\max }$ & 62 & Transmit power & $31.2 \mathrm{~mW}$ \\
\hline Simulation time & $500 \mathrm{~s}$ & Idle mode & $22.2 \mathrm{~mW}$ \\
\hline $\begin{array}{l}\text { Size of each } \\
\text { queue }\end{array}$ & 50 packets & Sleep state & $3 \mu \mathrm{W}$ \\
\hline$I_{\text {val }}$ & $\frac{C W_{\max }}{300}$ & Slot time & $9 \mu \mathrm{s}$ \\
\hline$C_{\min }$ & $40 \%$ & SIFS & $16 \mu \mathrm{s}$ \\
\hline$\alpha$ & 0.7 & $E_{T}$ & $0.001 \mathrm{~kJ}$ \\
\hline
\end{tabular}

(b) $\left(d_{i}<d_{i-1}\right)$. The observed delay in the decision interval $i$ is less than the delay in interval $i-1$, which indicates that the delay is gradually converging to $d_{\text {hop }}^{i}$. This prompts us to use the following multiplicative strategy to decrease the duty cycle cautiously:

$$
\delta_{i+1}=\delta_{i}\left(1-d_{i}\right)^{\tau_{i}} .
$$

The cautious decrease is an attempt to conserve node energy while maintaining the decreasing trend of average delay.

3. $(\eta==1)$. A value of $\eta$, equal to 1 , indicates that the observed per-hop delay is equal to the desired per-hop delay. This represents the ideal case, and the duty cycle remains unchanged.

\section{Performance evaluation}

In this section detailed performance analysis of $\mathrm{XL}$ WMSN is shown against three different layered protocol suites and a cross-layer protocol (PMAC). The schemes are simulated using network simulator NS-2 [24].
The network setup consists of 100 sensor nodes distributed in grid topology in a $100 \times 100 \mathrm{~m}$ field, as shown in Figure 2. The sink is positioned at $(70,40)$ coordinates, and the event region is centered at $(20,20)$ coordinates with a radius of $20 \mathrm{~m}$. The source nodes start event reporting after $10 \mathrm{~s}$ in the simulation time. All simulations are repeated five times and average results of simulations are presented. The network parameters are listed in Table 1. The layered protocol configurations for comparison include the following:

- $A O D V+S M A C$, where AODV [25] is a baseline-adaptive routing protocol, and SMAC [11] is the earliest and most straight forward duty cycle-based MAC protocol that incorporates local synchronization and static sleep listen cycles.

- $A O D V+T M A C$, where TMAC is proposed to enhance the poor results of the SMAC protocol under variable traffic load.

- $A O D V+$ Saxena, where Saxena MAC is based on CSMA-CA approach and aims to provide QoS for

Table 2 Qualitative comparison

\begin{tabular}{|c|c|c|c|c|c|c|}
\hline Protocol & Duty cycle & $\begin{array}{l}\text { Service } \\
\text { differentiation }\end{array}$ & $\begin{array}{l}\text { Priority } \\
\text { assignment }\end{array}$ & Traffic adaptive & $\begin{array}{l}\text { Energy } \\
\text { awareness }\end{array}$ & $\begin{array}{l}\text { Delay } \\
\text { awareness }\end{array}$ \\
\hline SMAC & $\begin{array}{l}\text { Static across all } \\
\text { nodes }\end{array}$ & No & No & No & No & No \\
\hline TMAC & Dynamic & No & No & Yes & No & No \\
\hline Saxena et al. & Dynamic & Yes & Yes & Yes & No & No \\
\hline PMAC & $\begin{array}{l}\text { Static for each } \\
\text { grade }\end{array}$ & No & No & No & No & Yes \\
\hline XL-WMSN & Dynamic & Yes & Yes & Yes & Yes & Yes \\
\hline
\end{tabular}




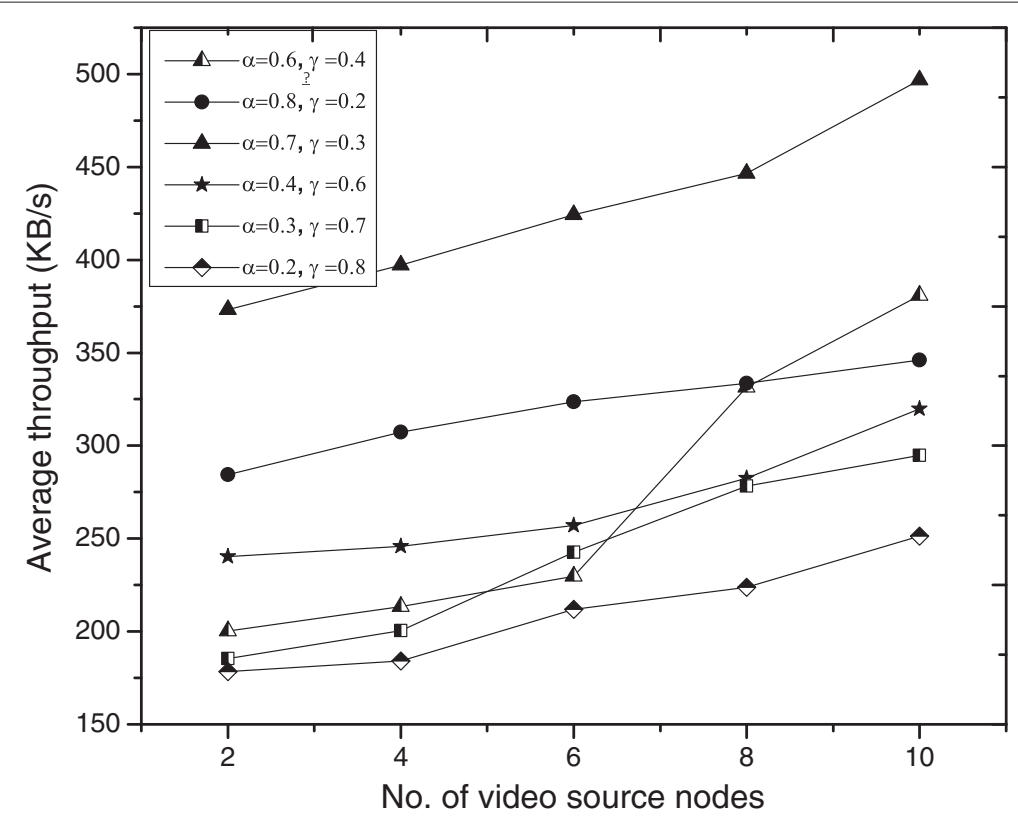

Figure 3 Average throughput for different weights for PST and utilization.

multimedia communications over sensor networks while conserving energy.

SMAC and TMAC are considered as baseline protocols used for comparison by majority of the duty cycle-based MAC protocols. PMAC has been chosen for comparison as it is a cross-layer MAC protocol which shares the same objective as XL-WMSN, i.e., reducing end-to-end delay. Saxena et al. uses a dynamic MAC protocol for WMSN, which is aimed at reducing delay and increasing throughput. Although multipriority queuing and duty cycle adaption are common features between Saxena et al.s protocol and XL-WMSN, we use a different approach to adjust the duty cycle.

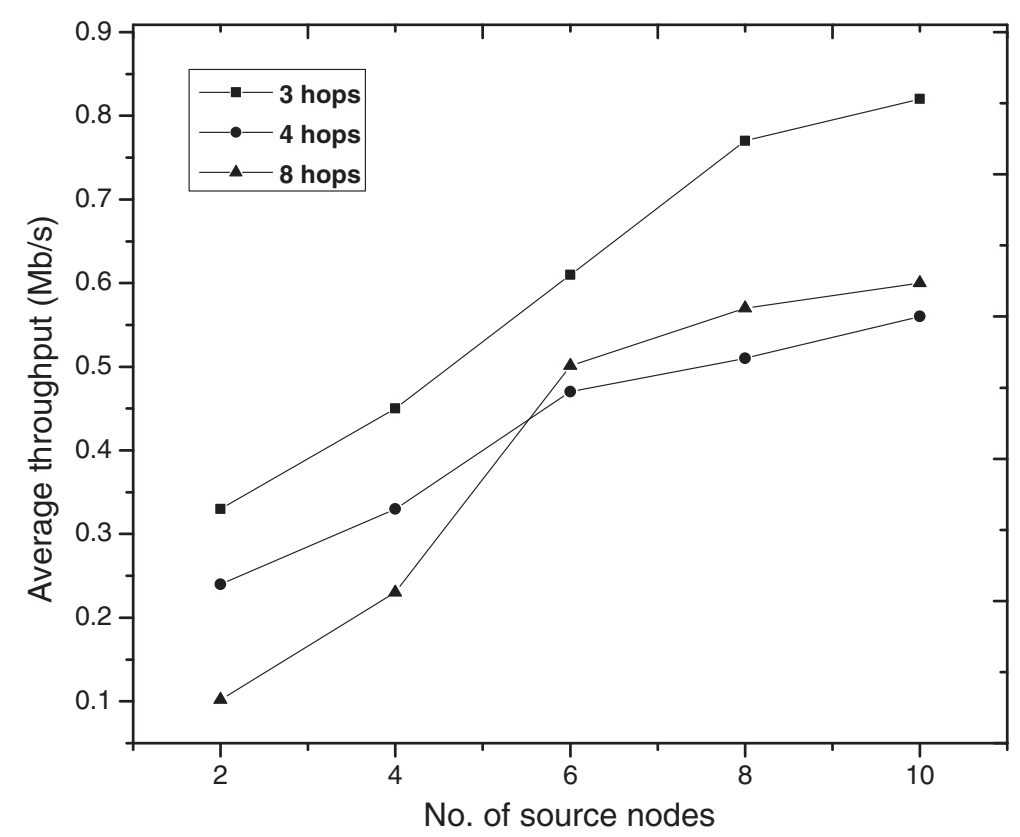

Figure 4 Average throughput with respect to hop count $(\delta)$. 
Saxena et al.s protocol has a fixed duty cycle for each traffic type, whereas the XL-WMSN duty cycle assignment scheme takes end-to-end delay deadline traffic types as well as traffic load into consideration. Furthermore, the DCA scheme is complemented by a delay-constrained routing protocol that helps to meet end-to-end delay deadlines. However, energy consumption is greater than in Saxena et al. because our duty cycle is variable and can grow until the maximum awake period, i.e., always awake. A qualitative comparison of these protocols is provided in Table 2. Ad hoc on-demand distance vector (AODV) is implemented with the following settings: there are maximum of three RREQ retries, route refresh time-out is $20 \mathrm{~s}$, and hello interval is $1,000 \mathrm{~ms}$.

There are various parameters that influence the design and performance of XL-WMSN, which include weight assignment and end-to-end delay deadline. Figure 3 presents the effect on average throughput of assigning different values to $\alpha$. As can be seen in the figure, throughput is higher for larger values of $\alpha$, which indicates that higher priority should be given to PST during route establishment. On the contrary, the throughput drops if $\alpha$ is greater than 0.7; this occurs because channel utilization is also an important parameter (to be considered) in the routing decision, and dropping its weight more than 0.3 will yield non-optimal results. Figure 4 presents the effect of hopcount $(\delta)$ on average throughput. Increasing the value of $\delta$ results in longer paths which reduces the probability of data delivery.
Another parameter that has an impact on the duty cycle is the end-to-end delay deadline. To determine this impact, the average duty cycle of all the nodes, on the routing path, within a distance of four hops from the sink is calculated. Four hops are chosen because there is higher contention near the sink, which provides a good opportunity to observe the behavior of duty cycle assignment. The average values are presented in Figure 5. The average duty cycle value in DCA decreases as the end-to-end delay deadline is relaxed from 0.1 to $0.7 \mathrm{~s}$. As can be seen, end-to-end delay deadline does not have any impact on SMAC and Saxena et al.s protocol, since their duty cycle assignment scheme is non-adaptive and independent of end-to-end delay requirement.

\subsubsection{Average throughput}

The average throughput comparison of XL-WMSN against other protocols is presented in this section. Throughput is the amount of data received at the destination within a specified amount of time. Figure 6a shows that XL-WMSN significantly outperforms the other protocols in terms of overall throughput. The average throughput of XL-WMSN is higher than Saxena MAC, SMAC, and TMAC. The SMAC and TMAC throughput is low because these protocols are not contention-aware and do not employ any service differentiation, resulting in collisions and high packet loss in high data rate networks. Figure $6 \mathrm{~b}$ further illustrates that XL-WMSN performs consistently better than other protocols, even with increasing packet arrival rate. This happens because

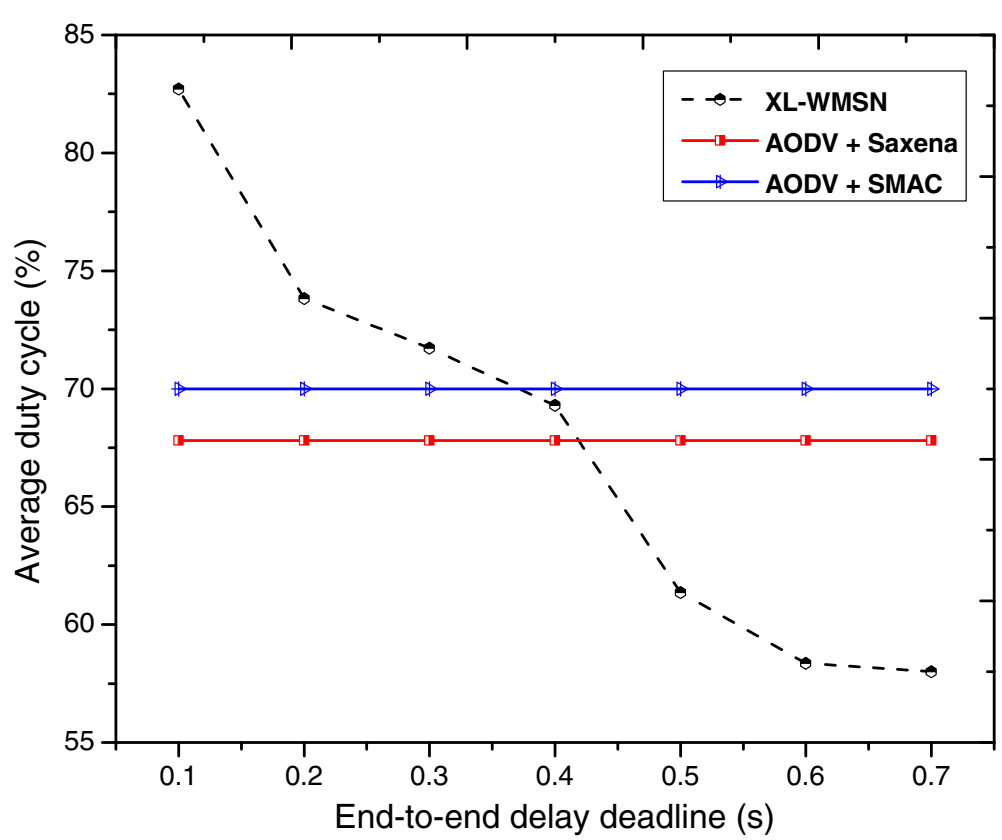

Figure 5 Average duty cycle comparison with respect to delay deadline. 

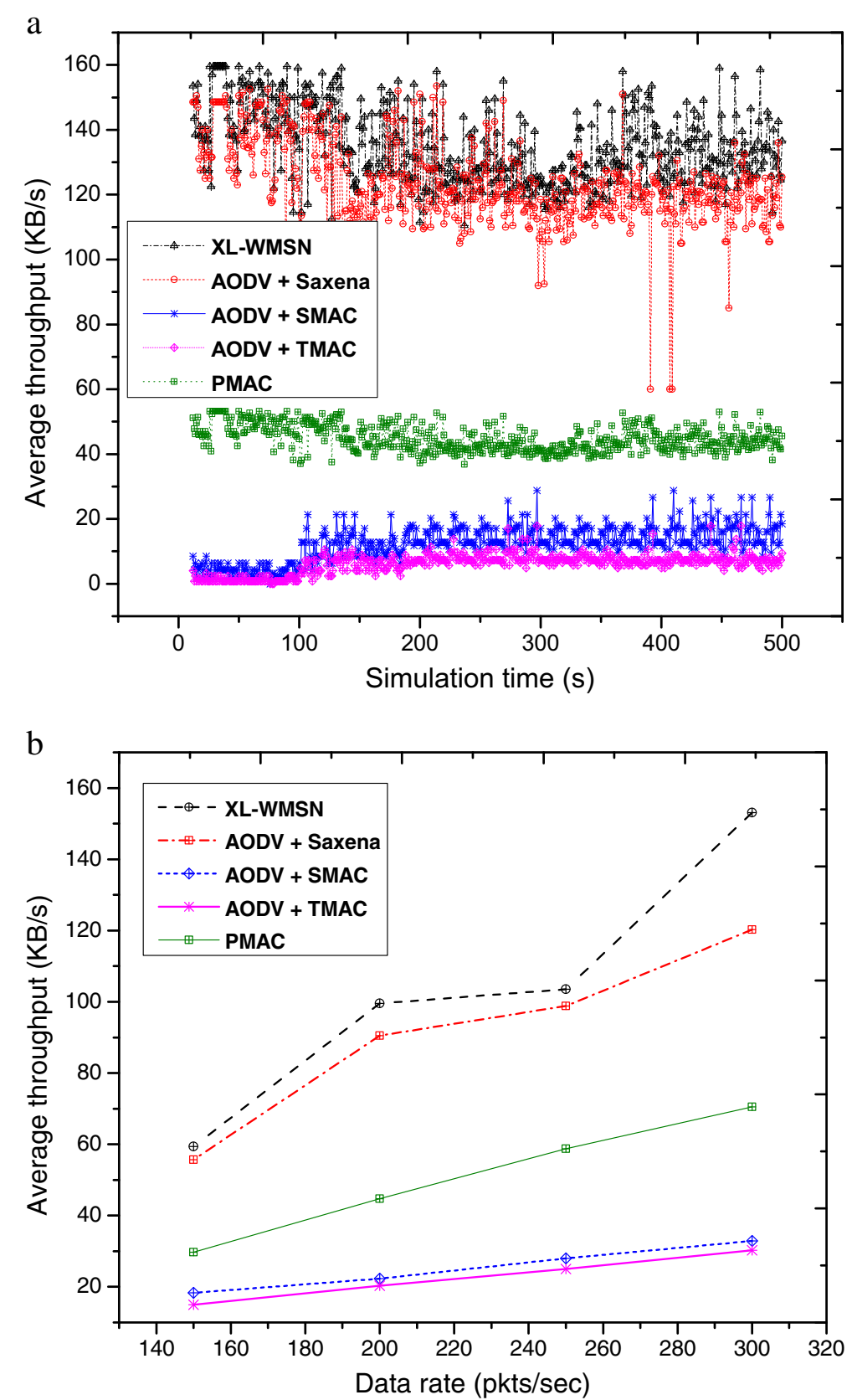

Figure 6 Average throughput. (a) Average throughput comparison with respect to simulation time. (b) Throughput comparison with respect to data rate.

XL-WMSN adapts duty cycle with increasing traffic rate and PST.

\subsubsection{Average end-to-end delay}

The end-to-end delay of a packet is measured as the time difference between the packet generation time and the time when it is received by the sink. Delays experienced by individual data packets are averaged over the total number of packets received by the sink. Figure 7a shows that XL-WMSN provides better overall delay than Saxena MAC, SMAC, and TMAC. This happens because XL-WMSN employs an adaptive duty cycle which adapts according to traffic rate as well as end-to-end deadline. Figure $7 \mathrm{~b}$ delineates the effect of increasing data rate on average end-to-end delay. As it shows, the average end-to-end delay increases with increase in packet arrival 
rate for all protocols, particularly where there is a sharp increase in SMAC and TMAC, with an increase in data rate. This occurs because these protocols have a uniform duty cycle which decreases their efficiency under variable traffic load, resulting in high latency. The average end-to-end delay of XL-WMSN and Saxena MAC increases gradually with an increase in data rate; however, the overall delay of XL-WMSN remains less than Saxena MAC. This is due to the reason that Saxena MAC adapts duty cycle according to traffic type only, whereas XL-WMSN adapts duty cycle according to traffic rate as well as end-to-end delay deadline of each traffic class.

Figure $8 \mathrm{a}, \mathrm{b}$ shows the average throughput and end-toend delay for a random topology. It is observed that the overall throughput in a random topology is less, but the general trend of throughput and delay for the different protocol suites is the same as in organized topology. XLWMSN has the best throughput and least delay compared to PMAC and Saxena et al.

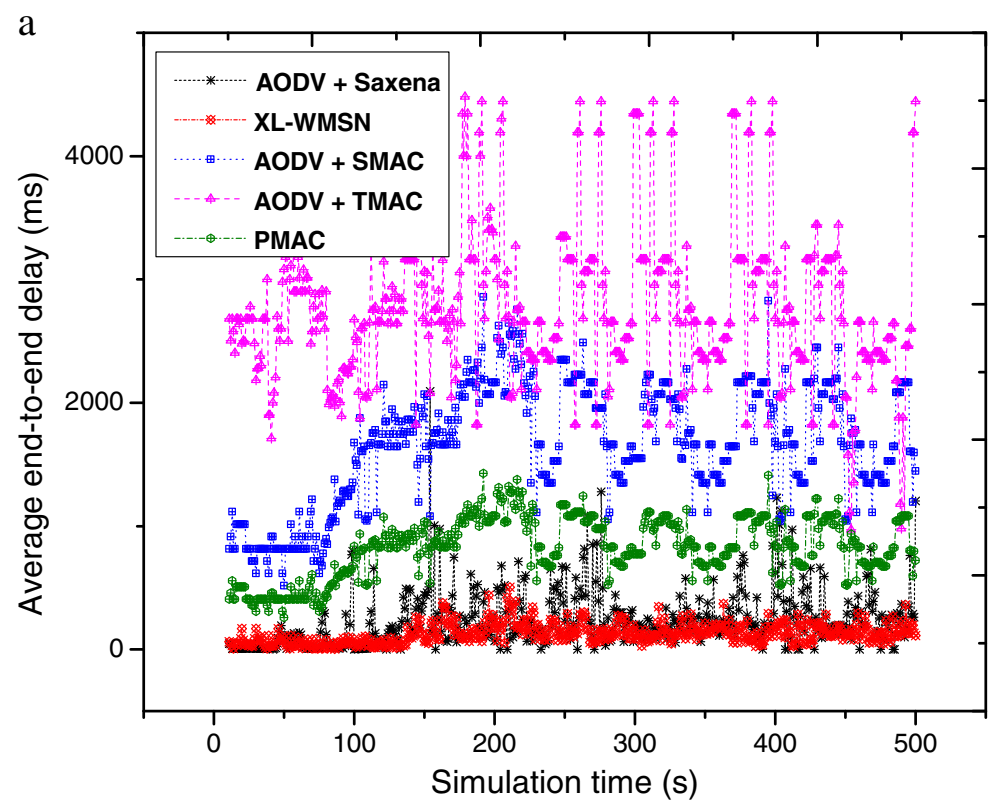

b

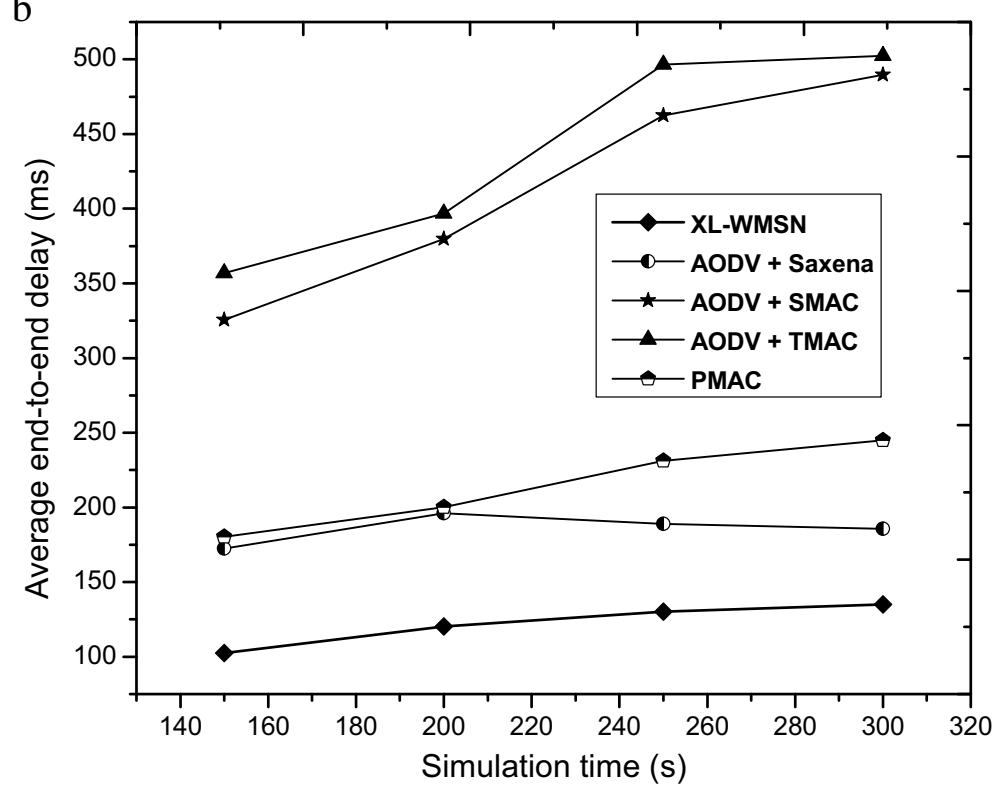

Figure 7 Average end-to-end delay. (a) Average end-to-end delay comparison with respect to simulation time. (b) Average end-to-end delay comparison with respect to data rate. 

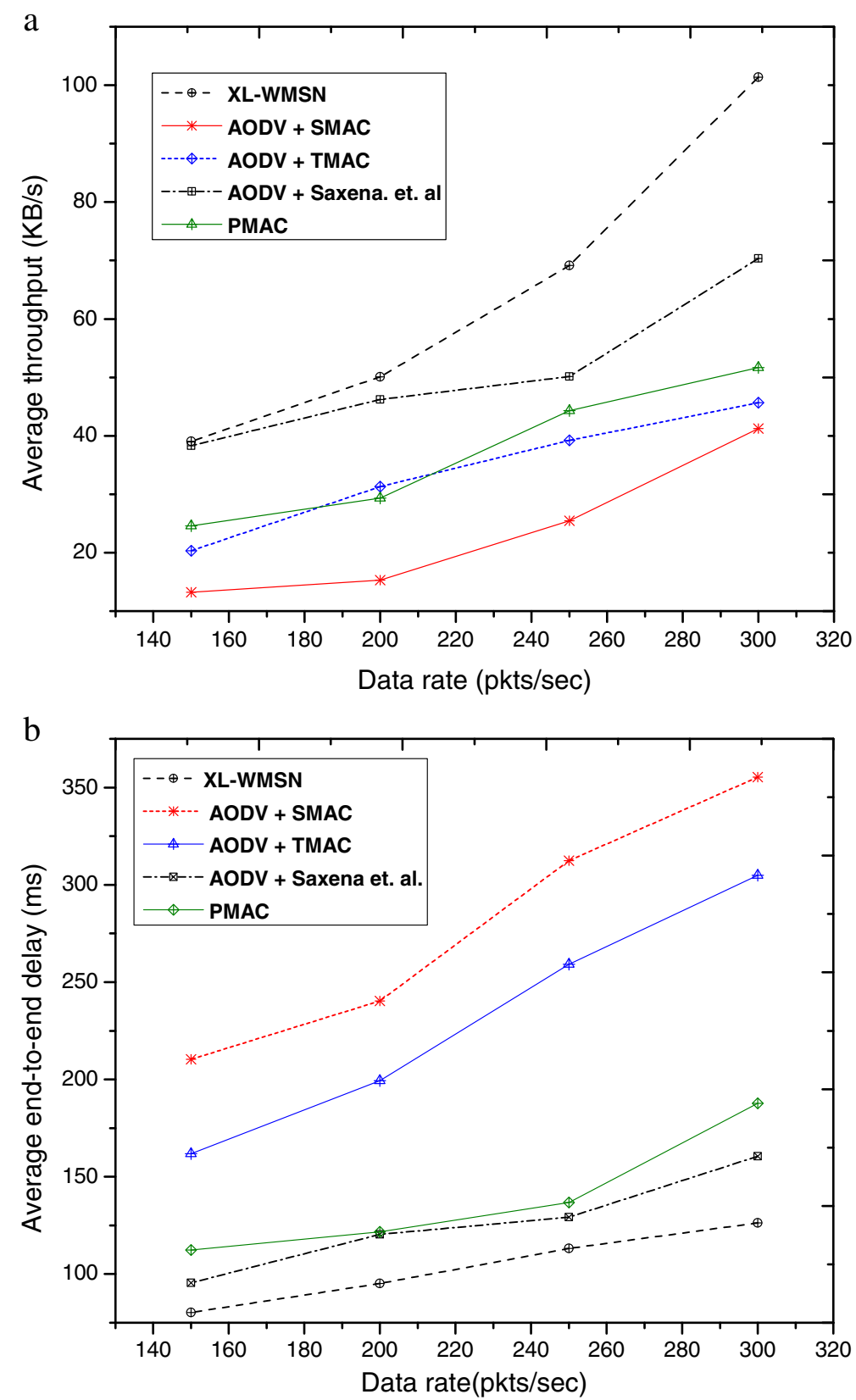

Figure 8 Average throughput and end-to-end delay in a random topology. (a) Average throughput comparison with respect to simulation time. (b) Average end-to-end delay comparison with respect to data rate.

\subsubsection{Energy}

Generally, a radio has four states: listen, receive, transmit, and sleep. The power consumption by each of these states can be symbolized as $P_{\mathrm{l}}, P_{\mathrm{r}}, P_{\mathrm{t}}$ and $P_{\mathrm{s}}$. We use the power values of Mica2 CC1000 radio [26], as shown in Table 1, for the actual representation of the model. The total energy consumption of a radio device can be measured by determining the amount of time it spends in each state denoted by $T_{\mathrm{l}}, T_{\mathrm{r}}, T_{\mathrm{t}}$ and $T_{\mathrm{s}}$.
Thus, the expected energy consumption of a node can be modeled as

$$
E=E_{\mathrm{l}}+E_{\mathrm{r}}+E_{\mathrm{t}}+E_{\mathrm{s}}=P_{\mathrm{l}} T_{\mathrm{l}}+P_{\mathrm{r}} T_{\mathrm{r}}+P_{\mathrm{t}} T_{\mathrm{t}}+P_{\mathrm{s}} T_{\mathrm{s}} .
$$

The total energy consumption, $E_{\text {Total, }}$ is modeled as

$$
E_{\text {Total }}=\sum_{i=0}^{n} P_{\mathrm{l}} T_{\mathrm{l}}^{i}+P_{r} T_{\mathrm{r}}^{i}+P_{\mathrm{t}} T_{\mathrm{t}}^{i}+P_{\mathrm{s}} T_{\mathrm{s}}^{i}
$$




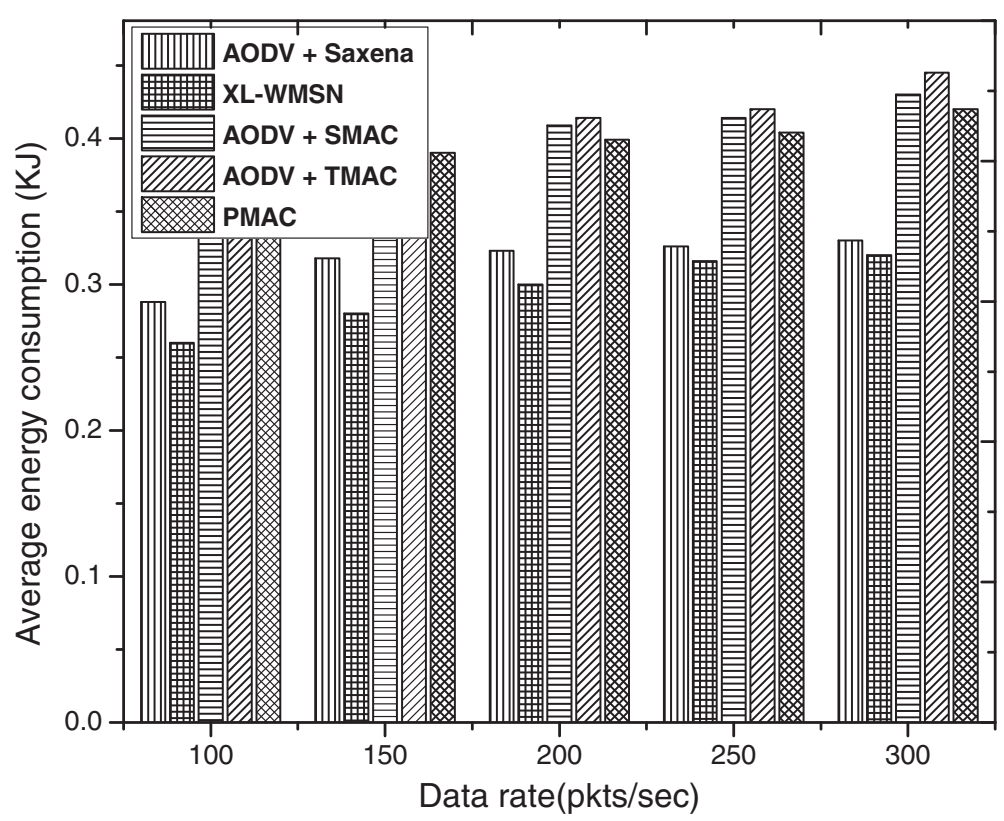

Figure 9 Average energy consumption comparison with respect to data rate.

where $n$ is the total number of nodes in the network. The average value of $E$ is taken after ten simulation runs. The total energy consumption is the sum of the energy consumption of all nodes in the network. Figure 9 illustrates the energy consumption of XL-WMSN in comparison to other protocols. The energy consumption of XL-WMSN is lesser than all protocols. This occurs because XL-WMSN employs energy-aware admission control and tries to avoid same path (by selecting alternative nodes with higher weights). Further, XL-WMSN has an adaptive MAC and can dynamically adjust the duty cycle according to traffic load, which prevents energy wastage due to idle listening, whereas Saxena employs a static duty cycle for each traffic flow. SMAC, TMAC, and PMAC do not provide any service differentiation; thus, they have uniform duty cycles, which lead to high-energy consumption.

\section{Conclusions}

The cross-layer paradigm for providing QoS guarantees like deadline-aware information delivery while conserving energy is the major focus of this research. A crosslayer approach is especially advantageous in WMSNs due to strict and often conflicting QoS requirements. In this work, XL-WMSN, a new cross-layer protocol for WMSNs is proposed. XL-WMSN consists of different components that work together to meet applicationspecific, end-to-end delay deadline. It consists of an energy-aware admission control policy that is aimed at increasing the lifetime of individual nodes in particular as well as the lifetime of the network as a whole. It includes delay- and channel-aware routing protocols which work towards choosing the least delay paths to deliver data from source to destination. The routing protocol utilizes PST and channel utilization information and a weighted cost function to choose most appropriate path. The proposed protocol consists of a prioritybased queuing mechanism that provides service differentiation between different traffic types. Lastly, XLWMSN includes a dynamic deadline-aware DCA scheme to reduce end-to-end latency during heavy traffic load and conserves energy during light traffic load. Performance comparisons with existing protocols have shown that XLWMSN provides better performance in terms of higher throughput and lower end-to-end delay as compared to well-known solutions like Saxena et al., PMAC, SMAC, and TMAC.

\section{Competing interests}

Both authors declare that they have no competing interests.

Received: 31 December 2012 Accepted: 4 June 2013

Published: 24 June 2013

\section{References}

1. F Liu, K Zhou, D Wang, in Proceedings of the Third Annual IEEE Communications Society on Sensor and Ad Hoc Communications and Networks, vol. 3. Application of video sensor networks in traffic surveillance (IEEE Piscataway, 2006), pp. 916-919

2. K Johannes, Wireless video sensor network and its application in digital zoo, Thesis, Umei University (2010) 
3. T Semertzidis, K Dimitropoulos, A Koutsia, N Grammalidis, Video sensor network for real-time traffic monitoring and surveillance. Intell. Transp. Syst. IET. 4(2), 103-112 (2010)

4. S Chen, K Nahrstedt, Distributed quality-of-service routing in ad hoc networks. Selected Areas Commun. IEEE J. 17(8), 1488-1505 (1999)

5. O Chipara, Z He, G Xing, Q Chen, X Wang, C Lu, J Stankovic, T Abdelzaher, in Proceedings of 14th IEEE International Workshop on Quality of Service, 2006. Real-time power-aware routing in sensor networks (IWQoS 2006, 19-21 June 2006), pp. 83-92

6. THe, JA Stankovic, L Chenyang, T Abdelzaher, in Proceedings of the 23rd International Conference on Distributed Computing Systems. SPEED: a stateless protocol for real-time communication in sensor networks (Macau, 19-22 May 2003), pp. 46-55

7. E Felemban, C-G Lee, E Ekici, MMSPEED: multipath multi-SPEED protocol for QoS guarantee of reliability and timeliness in wireless sensor networks. Mobile Comput. IEEE Trans. 5(6), 738-754 (2006)

8. K Akkaya, M Younis, Energy-aware delay-constrained routing in wireless sensor networks. Int. J. CommunSyst. 17(6), 663-687 (2004)

9. C Lu, BM Blum, TF Abdelzaher, JA Stankovic, T He, in Proceedings of the Eighth IEEE Real-Time and Embedded Technology and Applications Symposium. RAP: a real-time communication architecture for large-scale wireless sensor networks (IEEE San Jose, 2002), pp. 55-66

10. DG Costa, LA Guedes, A survey on multimedia-based cross-layer optimization in visual sensor networks. Sensors. 11(5), 5439-5468 (2011)

11. W Ye, J Heidemann, D Estrin, Medium access control with coordinated adaptive sleeping for wireless sensor networks. IEEE/ACM Trans. Netw. 12(3), 493-506 (2004)

12. TV Dam, K Langendoen, in Proceedings of the 1st International Conference on Embedded Networked Sensor Systems. An adaptive energy-efficient MAC protocol for wireless sensor networks (ACM Los Angeles, 2003), pp. $171-180$

13. N Saxena, A Roy, J Shin, Dynamic duty cycle and adaptive contention window based QOS-MAC protocol for wireless multimedia sensor networks. Comput. Netw. 52(13), 2532-2542 (2008)

14. GA Shah, W Liang, X Shen, in Proceedings of IEEE GLOBECOM 2010. Cross-layer design for QoS support in wireless multimedia sensor networks (Miami, 6-10 December 2010), pp. 1-5

15. T Melodia, IF Akyilidz, Cross-layer QoS-aware communication for ultra wide band wireless multimedia sensor networks. IEEE J. Selected Commun. 28, 653-663 (2010)

16. M Chen, V Leung, S Mao, M Li, in Proceedings of the IEEE Vehicular Technology Conference (VTC 08). Cross-layer and path priority scheduling based real-time video communications over wireless sensor networks (Marina Bay, May 2008), pp. 2873-2877

17. F Tong, R Xie, L Shu, Y-C Kim, A cross-layer duty cycle MAC protocol supporting a pipeline feature for wireless sensor networks. Sensors. 11(5), 5183-5201 (2011)

18. Z Hamid, F Bashir, JY Pyun, in Proceedings of Fourth International Conference on Ubiquitous and Future Networks (ICUFN 2012). Cross-layer QoS routing protocol for multimedia communications in sensor networks (Phuket, July 2012), pp. 498-502

19. Y Yang, R Kravets, Contention-aware admission control for ad hoc networks. Mobile Comput. IEEE Trans. 4(4), 363-377 (2005)

20. Y Pei, V Ambetkar, Distributed flow admission control for multimedia services over wireless Ad Hoc Networks. Wireless Personal Commun. 42(1), 23-40 (2007)

21. X Yin, X Zhou, M Pan, S Li, in Proceedings of the IEEE International Conference on Networking, Sensing and Control. Admission control with multi-constrained QoS providing in wireless sensor networks (Chicago, LL, 10-12 April 2010), pp. 524-529

22. G Anastasi, M Conti, M Di Francesco, A Passarella, Energy conservation in wireless sensor networks: a survey. Ad Hoc Netw. 7(3), 537-568 (2009)

23. I Demirkol, C Ersoy, F Alagoz, MAC protocols for wireless sensor networks: a survey. Commun. Mag. IEEE. 44(4), 115-121 (2006)

24. Information Science Institute, NS-2 network simulator, software package, (Information Science Institute, 2003). www.isi.edu/nsnam/ns. Accessed July 2012

25. CE Perkins, EM Royer, in Proceedings. WMCSA' 99 . Second IEEE Workshop on. Ad hoc on-demand distance vector routing," Mobile Computing Systems and Application,1999., (25-26 February 1999), pp. 90-100
26. W Ye, F Silva, J Heidemann, in Proceedings of the 4th International Conference on Embedded Networked Sensor Systems SenSys'06. Ultra-low duty cycle MAC with scheduled channel polling (Boulder, 1-3 November 2006), pp. 321-334

doi:10.1186/1687-1499-2013-174

Cite this article as: Hamid and Bashir: XL-WMSN: cross-layer quality of service protocol for wireless multimedia sensor networks. EURASIP Journal on Wireless Communications and Networking 2013 2013:174.

\section{Submit your manuscript to a SpringerOpen ${ }^{\circ}$ journal and benefit from:}

- Convenient online submission

- Rigorous peer review

- Immediate publication on acceptance

- Open access: articles freely available online

- High visibility within the field

- Retaining the copyright to your article

Submit your next manuscript at $\boldsymbol{~ s p r i n g e r o p e n . c o m ~}$ 